\title{
Transdisciplinary Design Aspects of an Air Mobile Stroke Unit
}

\author{
David CHIPPERFIELD ${ }^{\mathrm{a}, \mathrm{b}}$, Michael CHEESMAN ${ }^{\mathrm{a}, \mathrm{b}}$, Cees BIL ${ }^{\mathrm{a}, 1}$ and Greg HANLON ${ }^{\mathrm{b}}$ \\ ${ }^{a}$ RMIT University, School of Engineering, Melbourne, Australia \\ ${ }^{\mathrm{b}}$ Five Rings Aerospace Pty Ltd, Melbourne, Australia
}

\begin{abstract}
Stroke is highly treatable but time critical. The greatest opportunity to improve outcomes is in the first 'Golden Hour' after onset. Pre-hospital care for stroke in Australia is patchy and poorly coordinated, resulting in gross disparities in clinical outcomes between rural and urban Australians. Clinical outcomes are at least twice as poor for rural Australians compared to their urban counterparts. A proposed solution is an Air MSU, an aircraft configured for rapid response to stroke victims so that diagnosis and treatment can commence onsite. This concept follows the tradition of the Royal Flying Doctors Service who have been providing medical services to rural Australians since 1928. This paper discusses the conflicting medical and aerospace requirements for an aircraft equipped with a CT-scanner including supporting equipment and personnel.
\end{abstract}

Keywords. Stroke medical response, Golden Hour, air mobile stroke unit requirements

\section{Introduction}

Worldwide, there are over 6 million deaths annually due to stroke. In Australia, the number of strokes is anticipated to increase from 60,000 to 132,500 per year by 2050 . Stroke is highly treatable but time critical. The greatest opportunity to improve outcomes is in the first 'Golden Hour' after onset. Pre-hospital care for stroke in Australia is patchy and poorly coordinated, resulting in gross disparities in clinical outcomes between rural and urban Australians. The incidence of stroke is approximately $20 \%$ higher in rural areas, particularly for Indigenous Australians. Clinical outcomes are at least twice as poor for rural Australians compared to their urban counterparts. To optimise the prehospital treatment of stroke, a transformative research program is proposed to develop, test and implement novel disruptive technologies. The aim is to reduce morbidity and mortality, and narrow the urban, rural and Indigenous healthcare gaps. Part of this research program is the development of a Road and Air Mobile Stroke Unit (MSU) that can bring diagnostic and treatment equipment to the patient [1].

Stroke is a primary cause of death, disability, and dementia [2][3]. In the coming decades the prevalence of stroke is expected to increase because of the increase of the aged population in most countries [4]. Apart from considerable suffering for the patients and their families, stroke also results in enormous costs to society, costs associated with loss of work hours and with long-term care expenses [5][6].

\footnotetext{
${ }^{1}$ Corresponding Author, Mail: cees.bil@rmit.edu.au.
} 

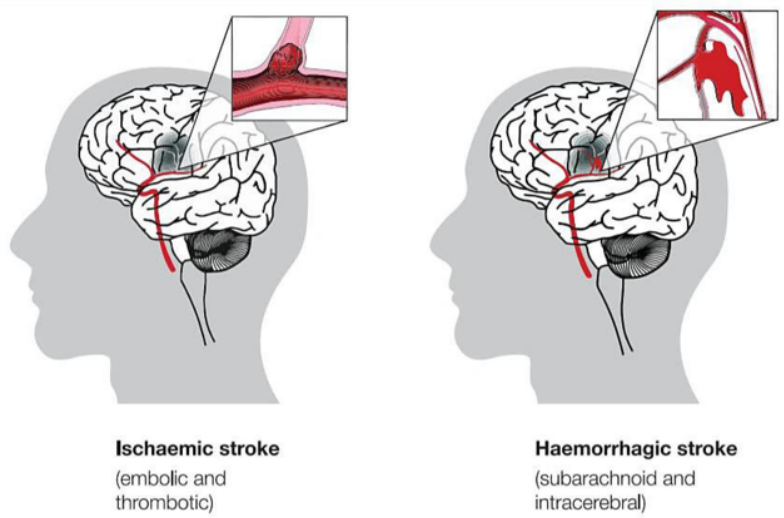

Figure 1. Ischaemic stroke and haemorrhagic stroke [2].

Two major forms of stroke can be distinguished (Figure 1). An ischaemic stroke is characterized by blocking (clots) of an artery transporting blood to parts of the brain. The second kind of stroke is the haemorrhagic stroke, caused by bursting of a blood vessel within the brain and floods the surrounding area. Due to the pressure in the artery, the rupturing blood can damage the sensitive brain tissue. Usually it is impossible to identify the stroke type on the basis of symptoms. Therefore, imaging tests of the brain are required to determine the stroke type and the extent of damage. Those scans can be performed by either a Computerised Tomography (CT) scan using X-rays for producing 2- or 3-dimensional images or by a similar Magnetic Resonance Imaging (MRI) scan which is based on generating a magnetic field and low-energy radio waves. In general, a CT is less sensitive than an MRI but is considered sufficient for the identification of an ischaemic stroke [7].

After the diagnosis of a stroke the patient should be treated as soon as possible to avoid further damageto the brain. Acute ischemic stroke is treatable by thrombolysis with recombinant tissue Plasminogen Activator (rtPA) or by removal of the clot in the brainsupplying vessel by Intra-Arterial Treatment (IAT) in case of Large Vessel Occlusion (LVO). Both types of recanalizing treatments must be delivered as quickly as possible after the onset of stroke symptoms because the chances of rescuing the ischemic brain from irreversible damage decline rapidly and even minutes of delay count [8][9]. Haemorrhagic stroke is treated by emergency surgery in order to remove the accumulated blood coming out of the ruptured vessel. Simultaneously the blood pressure needs to be reduced by using medication [9][10].

\section{Air Mobile Stroke Unit (Air MSU)}

Timely delivery of recanalizing stroke treatment is a serious medical problem, especially in countries with large remote areas and low population densities, in which coverage with specialized stroke centres is poor. Within a single country, the rural or urban setting and the associated distances to nearest stroke centre are key factors in the delivery of time-sensitive stroke treatment [11][12]. Reported times from symptom onset to admission to rural hospitals range from 5 hours to 30 hours [13][14]. In Australia, for example, the likelihood that patients in rural areas could access a stroke unit in time was only $3 \%$, whereas the likelihood for patients in metropolitan areas was $77 \%$ [15]. These 
delays before hospital admission are a major reason for low thrombolysis rates of $1 \%$ to $6 \%$ for stroke patients in rural areas in different countries [16-19]. Such a disparity in regard to acute stroke treatment is a major medical problem not only in low-income countries but also in middle- and high-income countries, such as Brazil, Russia, the United States, Canada, and Australia [13,16-19].

A recently developed concept is the provision of diagnosis and treatment of stroke at the emergency site. This is based on a Road Mobile Stroke Unit equipped with not only the conventional ambulance equipment, but with all of the tools necessary for prehospital diagnosis and treatment, and a rational triage decision with regard to most appropriate target hospital: A computed tomography (CT) scanner for multimodal imaging studies, a point-of-care (POC) laboratory for all blood tests required for treatment in accordance with approval criteria [20] and stroke management guidelines [21][22] and a telemedicine connection to hospital stroke experts.

Trials have shown that this approach significantly reduces delays before intravenous (IV) thrombolysis [23-26]. The rate of patients treated within $60 \mathrm{~min}$ after the onset of stroke symptoms ("Golden Hour") increased dramatically, from 4\% to 57\% [23]. This reduction in delay before treatment is due not only to a reduction in transport times but also to the marked improvement in efficiency of interfaces between the many groups of health care professionals involved in stroke management [27].

The Road MSU is suitable for metropolitan areas, but cannot provide the response time needed to reach people living in rural and remote areas. An alternative is the design of an Air MSU that is equipped specifically to respond to stroke victims living away from a suitable medical centre. Mobile CT-scanners currently available are intended for use in hospital settings and medical centres, not to fly on aircraft. CT-scanner operating requirements are generally not compatible with the environment of flying in an aircraft. In addition, special requirements must be met to provide sufficient clearance for patient and medical personnel to perform the scan and treat the patient. The scan will take place on the ground, potentially in hot, humid, wet or dusty conditions, while some treatment may be necessary in flight enroute to the hospital.

This paper discusses some of the design challenges associated with incorporating a standard CT-scanner with support equipment and personnel on an aircraft, with consideration for cabin ergonomic design and aircraft safety and operating requirements.

\section{Aeromedical Operations}

Aeromedical operations involve an aerial transport vehicle, either being a fixed wing aircraft or rotary wing aircraft (helicopter) for the purpose of medical operations. These operations allow for medical practitioners to treat patients in a timely manner and in more rural and hostile locations compared to conventional road ambulance means. The aircraft used for these operations are purpose equipped aircraft, fitted with all required items to successfully treat a patient and provide transfer to the nearest available medical centre or hospital.

The crew operating these aircraft are trained flight paramedics and provide comprehensive prehospital,emergency and critical care to all types of patients during aeromedical evacuation or rescue operations aboard. The type of operations being undertaken within the aeromedical field range from inter-hospital patient transfers, permitting the transport of patients between hospitals, to neonatal operations for the transport of young infants, to full scale search and rescue and trauma patient recovery 
from vehicular accidents. No one aircraft type is specifically suitable for all aeromedical operations, resulting in different size and type of aircraft being used.

The coordination and execution of aeromedical operations is generally conducted from a single resource centre. These centres rely on communication resources such as data and satellite to relay vital information between aircraft and ground crews. In some instances, multiple aircraft can be required for a single mission.

\subsection{Current Aeromedical Aircraft}

Within Australia there are several different aircraft operators serving the numerous states and territories. These operators maintain the various aircraft used to support the Aeromedical industry. There are several different aircraft types currently in use for aeromedical operations. These aircraft are sized and equipped based on the different locations and purposes. These aircraft fall into two categories: Fixed-Wing (aeroplane) and Rotary Wing (helicopter) aircraft. Table 1 and 2 list the primary fixed wing and rotary wing aircraft which

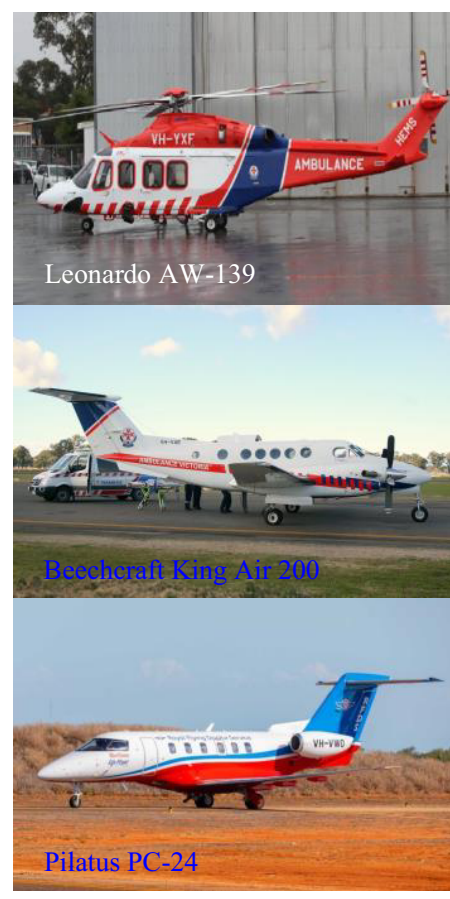

Figure 2. Some aircraft types in the Victorian Air Ambulance Service and Royal Flying Doctor Service fleet. are currently being utilised within Australian for aeromedical operations.

Table 1. Aeromedical Fixed Wing Aircraft.

\begin{tabular}{llccc}
\hline \multicolumn{1}{c}{ Manufacturer/Model } & \multicolumn{1}{c}{ Category } & $\begin{array}{c}\text { MTOW } \\
\text { kg }\end{array}$ & $\begin{array}{c}\text { Payload } \\
\text { kg }\end{array}$ & $\begin{array}{c}\text { Range } \\
\text { km }\end{array}$ \\
\hline Bombardier Challenger 604 & Jet Medium & 21,909 & 2,189 & 7,628 \\
Bombardier Learjet 45 & Jet Medium & 9,318 & 959 & 3,645 \\
Pilatus PC-24 & Jet Small & 8,318 & 1,136 & 3,611 \\
Beechcraft King Air 200 & Turboprop & 5,682 & 841 & 2,759 \\
& Medium Multi & & & \\
Beechcraft King Air 350 & Turboprop & 6,818 & 1,189 & 2,871 \\
Pilatus PC-12 & Medium Multi & 4,750 & 1,125 & 3,074 \\
\hline
\end{tabular}

Table 2. Aeromedical Rotary Wing Aircraft.

\begin{tabular}{llccc}
\hline \multicolumn{1}{c}{ Manufacturer/Model } & \multicolumn{1}{c}{ Category } & $\begin{array}{c}\text { MTOW } \\
\text { kg }\end{array}$ & $\begin{array}{c}\text { Payload } \\
\text { kg }\end{array}$ & $\begin{array}{c}\text { Range } \\
\text { km }\end{array}$ \\
\hline Airbus BO 105 & Light Multi & 2,605 & 896 & 437 \\
Airbus BK 117B2 & Med Multi & 3,357 & 1126 & 441 \\
Airbus H175 & Heavy Multi & 7,515 & 2,582 & 722 \\
Airbus H145 & Med Multi & 3,708 & 1,559 & 620 \\
Bell 412EP & Med Multi & 5,409 & 1,831 & 582 \\
Leonardo AW139 & Heavy Multi & 6,414 & 2,173 & 1,074 \\
Leonardo AW189 & Heavy Multi & 8,317 & 3,359 & 870 \\
Sikorsky S-92A & Heavy Multi & 12,045 & 3,371 & 810 \\
\hline
\end{tabular}




\subsection{Equipment Details}

For effective patient treatment upon arrival, the Air MSU must accommodate all necessary medical equipment and personnel for diagnosis and follow-up treatment. The most critical item is the CT-scanner that takes "slices" of X-ray images, thus creating a $3 \mathrm{D}$ image of the brain. The image quality must be such that it shows vascular occlusion or blood perfusion to determine the cause of the stroke and its location. Mobile CTscanners are designed to operate in a hospital environment and are not intended to be installed on aircraft. This is by far the most significant engineering challenge as CTscanners are heavy, typically between $600 \mathrm{~kg}$ to $800 \mathrm{~kg}$, and sensitive to vibration, external loads and environmental conditions.

The supporting medical equipment generally installed includes items to assist with patient monitoring and ventilation. These items are stowed in suitable mission cabinets and consoles throughout the cabin for efficient treatment. Table 3 lists the payload requirements for a typical Air MSU aircraft. The weight of installed equipment + crew + personnel is approximately $2,000 \mathrm{~kg}$.

Table 3. Medical Equipment List.

\begin{tabular}{l}
\hline \multicolumn{1}{c}{ Component } \\
\hline CT scanner system \\
CT scanner system batteries \\
Stabilization system \\
Telemedicine equipment \\
Computer Systems \\
Stretcher System \\
Stretcher System Restraint \\
Contrast medium injector \\
Heater/fridge \\
Technical Equipment Rack \\
Mission Console \\
Medical equipment (various) \\
Laboratory unit \\
Crew Seating (Standard) \\
Crew Seating (Swivel) \\
\hline
\end{tabular}

All the medical equipment listed is required to be stowed in accordance with load requirements as outlined by the aircraft certification. Accordingly, the utilisation of medical cabinets and consoles are necessary to stow loose items of mass. For larger specialised items, such as the CT scanner system, a bespoke load restraint system will be required. These types of systems will allow for the integration to the aircraft floor and cargo provisions and can incorporate provisions such as vibration suppression and stabilisation for the scanner, or patient turn tables and lifts for the stretcher systems.

\subsection{CT Scanner System}

For on-site treatment, a CT-scanner is required to help determine the size, location and type of stroke. A mobile CT-scanner is powered by either integrated rechargeable batteries or by an external power source. It can perform five to six scans or can be on standby mode for at least 8 hours. In-flight charging of the batteries can also be considered to extend the system standby time when not located at the base of operations. A tablet used for controlling and configuring the CT scanner is installed, however the system can also be operated remotely for operator safety and convenience. This is important in the Air MSU as cabin space can be restrictive.

The system has an operational temperature range of $10{ }^{\circ} \mathrm{C}$ to $40{ }^{\circ} \mathrm{C}$. This range allows for operations in some of the more remote areas across the country. To assist, the aircraft on-board environmental control system (air conditioner), will ensure the system remains within the required operational temperature range.

\subsection{Telemedicine Systems}

To aid in the treatment and help in the preparation of hospital admission, tele-medical systems are installed to provide near real-time communications and data transfer between 
the in-field paramedics and the hospital-based neurologist at a Comprehensive Stroke Care Centre. These systems enable real-time digital transmission of the imaging and physiological data to ensure both the correct treatment can be administered on-site, but also provide the receiving hospital with the correct information to continue patient treatment upon arrival. The telemedicine systems currently operate on the $4 \mathrm{G}$ cell band for data transmission. In the event of a mission occurring outside of usable cell range, the system can convert to satellite communications to relay all required data.

\subsection{Stretcher Systems}

To work in conjunction with the MSU CT-scanner system, a suitable stretcher is required. The stretcher supports the patient during the scanning operations and, in the event of inter-hospital transfer, secure the patient in the aircraft or ground vehicle. Currently, in Road MSUs a specialised Stryker PowerPro XT stretcher is used, shown in Figure 3. This system provides a combination of flexibility and ruggedness for operations in all expected mission environments. The stretcher has adjustable height to suit the CT scanner. This is achieved with the integral

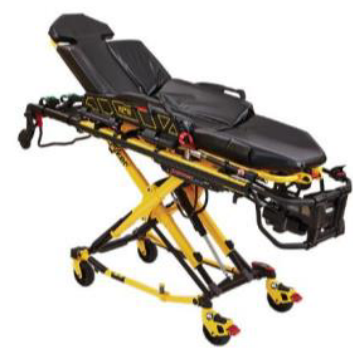

Figure 3. Stryker PowerPro XT hydraulic systems to permit the adjustment with zero-lift for operator safety.

\subsection{Medical Crew Seating}

During flight, the crew must be in a seated position. There are several crew seating options for integration into the aircraft. For treatment of the patient during flight, a purpose multi-mission seat is required. These seat types allow for translation and rotation. This provides the greatest flexibility to allow the crew to perform their required tasks. For Air MSU operations, specialist personnel are required to conduct specialist tasks and treatment. Seating provisions must be provided for the following medical crew:

(a) Paramedic

(b) Radiographer

(c) Nurse

(d) General practitioner or neurologist

(e) Second paramedic (highly desirable)

(f) Patient family (highly desirable)

\section{Project Specific Requirements}

\subsection{Crew Requirements}

Aeromedical operations are considered a specialised task within the industry. The crew who will be operating the medical systems will need to be fully trained and aware of these requirements when operating both in and around the aircraft. Further, crew will need to be trained on cabin layout and equipment operations to ensure they can perform their functions in a safe and efficient manner. 


\subsection{Landing Site}

Potential landing sites within the region that are suitable for an Air MSU will need to be considered. The landing sites will need to account for the overall length, and maximum landing weight of the aircraft. An Air MSU based at a metropolitan hub will allow for the utilisation of the existing resources at both aeromedical operators and Stroke Care Centres who currently operate a Road MSU. This configuration of facilities will require some additional logistical capabilities to transfer the patient from the airfield to the hospital. Further to this, the airfield can support $24 \mathrm{hr}$ operations. This will allow for the use of ground power facilities to provide power to the CT scanners when not in use.

\subsection{Vibration}

The cabin vibration will form the major design point for the integration of the proposed CT scanner into the aircraft. Given aircraft, specifically rotary wing aircraft, are prone to heavy vibrations compared to road base vehicles, some form of isolation system will be required to protect the sensitive equipment. The levels of vibration in terms of both frequency and amplitude will vary for the selected Air MSU aircraft. Further, as vibration is a function of mass, a simple analysis of an empty aircraft will be insufficient to analyse the installation. Several test flights would be required:

(a) Bare cabin to determine a baseline vibration spectrum for the aircraft. The data captured can be used in conjunction with equipment providers to assess the suitability of the environmental conditions and determine any isolation requirements.

(b) Operations with a representative mass that mimics the CT scanner and equipment. This will allow the isolation system to be fine-tuned prior to operating with the scanner installed, thus preventing damage to the equipment. This will also provide a better understanding of the cabin configuration and weight and balance limitations with all items installed.

(c) Operations with the full system installation. This will provide a final in-flight test of the system to ensure the vibration isolation is adequate to protect the system.

Further to test (c), the vibration system can remain installed to provide continued vibration monitoring to ensure the system does not exceed any limits. This vibration system may also form part of the final restraint of the system for the CT Scanner.

\subsection{System Installation}

The aircraft must be certified to the latest crash requirements of FAR 25.561 (fixed wing), and FAR 29.561 (rotary wing) for restraining items of mass within a cabin. The intention of these requirements it to protect the aircraft occupants from injury in the event of an emergency landing. A breakdown of the load requirements is outlined in Table 4. As such, the CT scanner will need to be restrained to loads of up to $20 \mathrm{~g}$ in some directions. The integration of the system into the aircraft will require some form of interface to secure the CT scanner to the aircraft floor and roof structure. Extensive analysis of both the scanner (including interface) and the aircraft will be required. While these design loads are high, it should be noted that corresponding requirements for installation within a road ambulance (AS/NZ4535:1998) are greater in some orientations. 
Therefore, should a certification program be undertaken for the CT scanner, the data generated will be suitable across both platforms allowing for integration into a road fleet as well.

Table 4. Critical Design Load Factors.

\begin{tabular}{lccc}
\hline Direction & FAR 25.561 Amdt 91 & FAR 29.561 Amdt 38 & AS/NZ4535:1998 \\
\hline Forward & $9.0 \mathrm{~g}$ & $16.0 \mathrm{~g}$ & $20.0 \mathrm{~g}$ \\
Downward & $6.0 \mathrm{~g}$ & $20.0 \mathrm{~g}$ & - \\
Sideward & $4.0 \mathrm{~g}$ & $8.0 \mathrm{~g}$ & $10.0 \mathrm{~g}$ \\
Upward & $3.0 \mathrm{~g}$ & $4.0 \mathrm{~g}$ & - \\
Rearward & $1.5 \mathrm{~g}$ & $1.5 \mathrm{~g}$ & - \\
\hline
\end{tabular}

The restraint of the patient and stretcher system will also need to be carefully considered. The patient it required to be restrained in the same manner as the seated occupants. Like with the scanner, a specialised interface may be required to permit translation of the occupant and stretcher to interface into the scanner.

\subsection{System Loading}

The operation of the system in terms of loading both equipment and patient will also be a critical factor. Given the size and weight of the scanner, special consideration will be required with developing the interface system. The need for translation along the cabin and forklift provisions will all need to be considered to allow for maintenance and reconfiguration of the aircraft. In conjunction with the loading of the scanner, patient loading, and positioning will also need to be considered. The patient will be required to be loaded firstly onto a stretcher and then into the aircraft. A typical Air MSU layout for the S-92A is shown in Figure 4.

With the safety culture in the medical industry, there has been a shift towards zero-lift operations. This safety element is a critical a part of the integration of the stretcher and scanner into the aircraft as these items must be loaded and secured with minimal operator lifting. The use of equipment such as stretcher loaders (Stryker Powerload) will be required for the patient loading phase of the mission, while items such as forklifts or cranes will be required for maintenance purposes to load/unload the scanner.

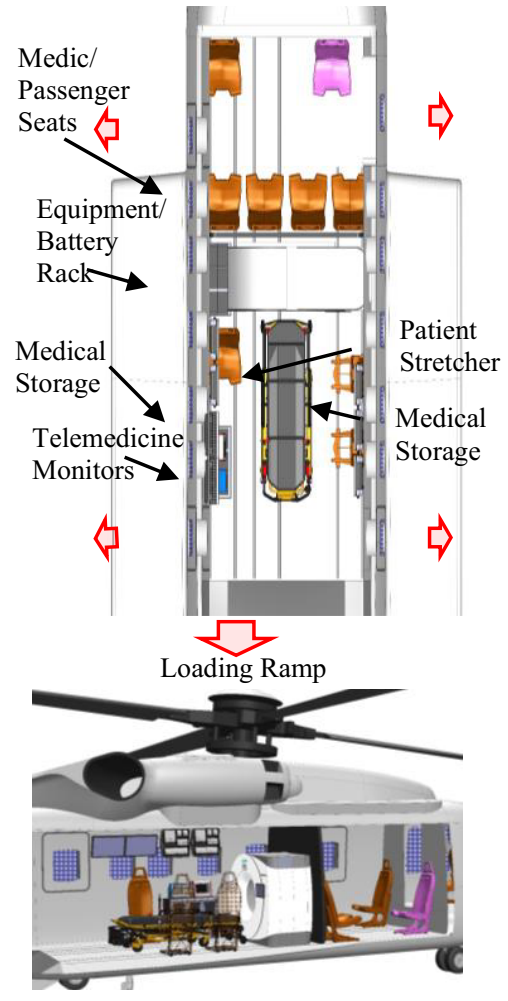

Figure 4. Typical Air MSU layout.

\subsection{Payload-Range Performance}

The main objective of the Air MSU is to provide diagnosis and begin treatment on a stroke victim within the first "golden hour". The Sikorsky S-92A can lift at least 3,000 $\mathrm{kg}$ of payload with sufficient fuel for 60 minutes of flight. This analysis includes a 30minutes fuel reserve, but excludes time and fuel used during taxi, take-off and landing. 
This can be increased by $544 \mathrm{~kg}$ with a Gross Weight Increase. With a payload of 2,000 $\mathrm{kg}$, the performance is improved to 60 minutes operations without re-fuelling. The payload-range performance is shown in Figure 5.
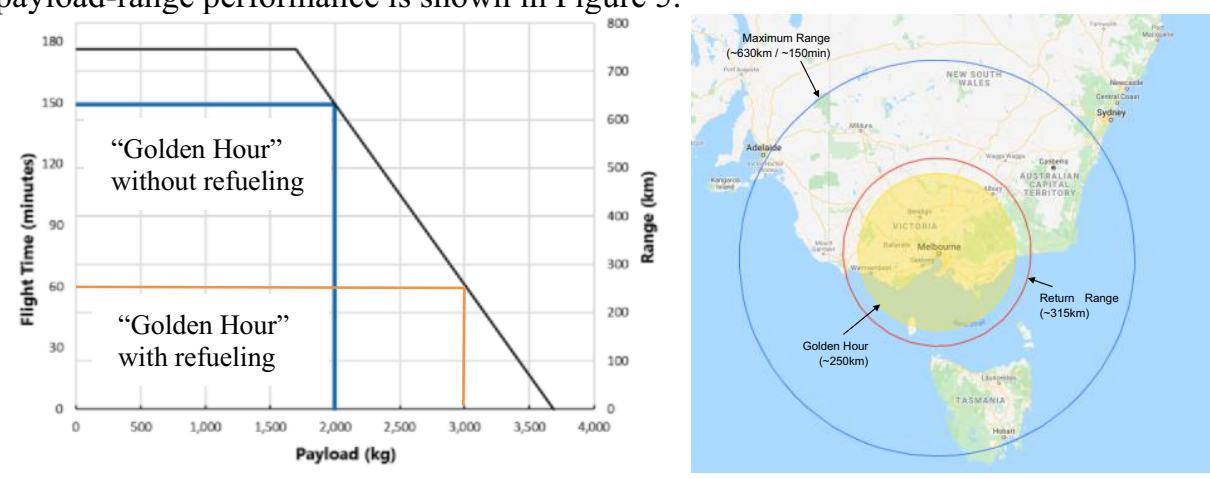

Figure 5. S-92A Air MSU payload versus range performance.

The proposed base for Air MSU operations is Essendon Airport in Melbourne, which is used as an aeromedical base for Air Ambulance Victoria, for fixed-wing aircraft and helicopters. With the cruise speed for the Sikorsky S-92A of between $252 \mathrm{~km} / \mathrm{h}$ and $280 \mathrm{~km} / \mathrm{h}$, the capable range of $250 \mathrm{~km}$ can be achieved in one hour, the Golden Hour.

\section{Conclusion}

An Air MSU is proposed that can reach stroke victims in rural and remote areas as quickly as possible to allow for diagnosis and treatment to commence onsite. The design of such a solution requires close interaction between medical personnel, aerospace engineers and medical equipment manufacturers to ensure a vehicle can be designed for efficient and effective operations. The major engineering challenge is the CT-scanner which are not designed to be installed on an aircraft. They are relatively heavy and sensitive to loads, vibrations and environmental changes. This means that, in collaboration with manufacturers, a parallel effort is made to reduce scanner weight without affecting its functionality. Alternatively, new technologies are in development that would make CT-scanners more portable. The third stakeholder are the medical personnel that need to work in a confined environment and require ready access to the patient, equipment, and medical supplies. This paper presented an overview of requirements that must be considered from a medical and aerospace engineering perspective to ensure safe and reliable operation.

\section{References}

[1] C. Bil, S. Walter, J. Sauer and S. Feldmann, Towards an air mobile stroke unit for rapid medical response in rural Australia, Advances in Transdisciplinary Engineering, 2019, Vol. 10, pp. 465-474.

[2] Ovbiagele B, Goldstein LB, Higashida RT et al., American Heart Association Advocacy Coordinating Committee and Stroke Council. Forecasting the future of stroke in the United States: a policy statement from the American Heart Association and American Stroke Association, Stroke, 2013, 44:2361-75.

[3] Writing Group Members, Mozaffarian D, Benjamin EJ, Go AS et al; American Heart Association Statistics Committee; Stroke Statistics Subcommittee. Heart disease and stroke statistics-2016 update: a report from the American Heart Association, Circulation, 2016; 133:e38-360. 
[4] V.L. Feigin, B. Norrving, G.A. Mensah, Global burden of stroke, Circulation Research, 2017;120:439448.

[5] J. Olesen, A. Gustavsson, M. Svensson, H.U. Wittchen, B. Jönsson, CDBE2010 study group; European Brain Council. The economic cost of brain disorders in Europe, Eur J Neurol, 2012, 19:155-62.

[6] V.L. Roger, A.S. Go, D.M. Lloyd-Jones et al; American Heart Association Statistics Committee and Stroke Statistics Subcommittee. Heart disease and stroke statistics--2012 update: a report from the American Heart Association. Circulation, 2012, 125:e2-220.

[7] Garcia-Esperon, NSC 2017, Stroke, Brain Foundation, viewed 14.10.2018, https://brainfoundation.org.au/disorders/stroke.

[8] J. Emberson, K.R. Lees, Lyden P et al; Stroke Thrombolysis Trialists' Collaborative Group. Effect of treatment delay, age, and stroke severity on the effects of intravenous thrombolysis with alteplase for acute ischaemic stroke: a meta-analysis of individual patient data from randomised trials, Lancet, 2014, 384:1929-35.

[9] J.L. Saver, Time is brain-quantified, Stroke, 2006, 37:263-6.

[10] A. Mandal, 2018 Stroke Treatment, News-Medical.net, 15.10.2018, <https://www.newsmedical.net/health/Stroke-Treatment.aspx>.

[11] A.M. Cox, C. McKevitt, A.G. Rudd and C.D. Wolfe, Socioeconomic status and stroke, Lancet Neurol, 2006, 5:181-8.

[12] Y. Teuschl and M. Brainin Stroke education: discrepancies among factors influencing prehospital delay and stroke knowledge, Int J Stroke, 2010, 5:187-208.

[13] J. Joubert, L.F. Prentice, T. Moulin et al., Stroke in rural areas and small communities, Stroke, 2008, 39:1920-8.

[14] J. Oliveira-Filho, S.C. Martins, O.M. Pontes-Neto et al; Executive Committee from Brazilian Stroke Society and the Scientific Department in Cerebrovascular Diseases. Guidelines for acute ischemic stroke treatment: part I., Arq Neuropsiquiatr, 2012; 70:621-9.

[15] A. Eissa, I. Krass, B.V. Bajorek, Optimizing the management of acute ischaemic stroke: a review of the utilization of intravenous recombinant tissue plasminogen activator (tPA), J Clin Pharm Ther, 2012, 37:620-9.

[16] D.A. Cadilhac, T. Purvis, M.F. Kilkenny et al; New South Wales Strokes Services Coordinating Committee; Agency for Clinical Innovation. Evaluation of rural stroke services: does implementation of coordinators and pathways improve care in rural hospitals? Stroke, 2013, 44:2848-53.

[17] L.V. Stakhovskaia, K.V. Shekhovtsova, T.I. Rozhkova et al., Medical service to patients with stroke at the prehospital stage in different cities of the Russian Federation, Zh Nevrol Psikhiatr Im S S Korsakova, 2010, 110:36-41.

[18] A. Ganesh, M. Camden, P. Lindsay et al; Canadian Stroke Audit Group. The quality of treatment of hyperacute ischemic stroke in Canada: a retrospective chart audit, CMAJ Open, 2014; 2:E233-9.

[19] J. Newbury, T. Kleinig, J. Leyden et al. Stroke Epidemiology in an Australian Rural Cohort (SEARCH). Int J Stroke, 2017, 12:161-8.

[20] Boehringer Ingelheim Limited. Actilyse. Summary of Product Characteristics, 2017. www.medicines.org.uk/emc/medicine/308. September 26, 2017.

[21] E.C. Jauch, J.L. Saver, H.P. Adams Jr et al; American Heart Association Stroke Council; Council on Cardiovascular Nursing; Council on Peripheral Vascular Disease; Council on Clinical Cardiology. Guidelines for the early management of patients with acute ischemic stroke: a guideline for healthcare professionals from the American Heart Association/American Stroke Association, Stroke, 2013, 44:870947.

[22] European Stroke Organisation (ESO) Executive Committee; ESO Writing Committee. Guidelines for management of ischaemic stroke and transient ischaemic attack 2008, Cerebrovasc Dis, 2008, 25:457507.

[23] S. Walter, P. Kostopoulos, A. Haass et al. Diagnosis and treatment of patients with stroke in a mobile stroke unit versus in hospital: a randomised controlled trial, Lancet Neurol, 2012, 11:397-404.

[24] A. Itrat, A. Taqui, R. Cerejo et al., Cleveland Pre-Hospital Acute Stroke Treatment Group. Telemedicine in prehospital stroke evaluation and thrombolysis: taking stroke treatment to the doorstep, JAMA Neurol, 2016, 73:162-8.

[25] M. Ebinger, B. Winter, M. Wendt et al., STEMO Consortium. Effect of the use of ambulance-based thrombolysis on time to thrombolysis in acute ischemic stroke: a randomized clinical trial, JAMA, 2014, 311:1622-31.

[26] R. Bowry, S. Parker, S.S. Rajan et al., Benefits of stroke treatment using a Mobile Stroke Unit compared with standard management: the BEST-MSU Study Run-In Phase, Stroke, 2015, 46:3370-4

[27] K. Fassbender, J.C. Grotta, S. Walter, I.Q. Grunwald, A. Ragoschke-Schumm and J.L. Saver, Mobile stroke units for prehospital thrombolysis, triage, and beyond: benefits and challenges, Lancet Neurol, 2017, 16:227-37. 